\title{
Almost sure-sign convergence of Hardy-type Dirichlet series
}

\author{
Daniel Carando* $\quad$ Andreas Defant ${ }^{\dagger} \quad$ Pablo Sevilla-Peris ${ }^{\ddagger}$
}

\begin{abstract}
Hartman proved in 1939 that the width of the largest possible strip in the complex plane, on which a Dirichlet series $\sum_{n} a_{n} n^{-s}$ is uniformly a.s.-sign convergent (i.e., $\sum_{n} \varepsilon_{n} a_{n} n^{-s}$ converges uniformly for almost all sequences of signs $\varepsilon_{n}= \pm 1$ ) but does not convergent absolutely, equals $1 / 2$. We study this result from a more modern point of view within the framework of so-called Hardy-type Dirichlet series with values in a Banach space
\end{abstract}

\section{Introduction}

The natural domains of convergence of Dirichlet series are half-planes. Given a Dirichlet series $D=$ $\sum a_{n} n^{-s}$ there are three abscissas which define the biggest half-planes on which $D$ converges, converges uniformly and converges absolutely:

$$
\sigma_{c}(D) \leq \sigma_{u}(D) \leq \sigma_{a}(D) .
$$

Whereas it is not difficult to show that

$$
\sup _{D \text { Dir. ser. }} \sigma_{a}(D)-\sigma_{u}(D)=1
$$

the main issue in the 1910's was to decide what the maximal width of the band on which a Dirichlet series can converge uniformly but not absolutely is. This problem was first considered by Harald Bohr and consists on computing the following number

$$
S:=\sup _{D \text { Dir. ser. }} \sigma_{a}(D)-\sigma_{u}(D) .
$$

Bohr himself [8] showed in 1913 that $S \leq 1 / 2$, but it was not until 1931 that Bohnenblust and Hille [7] proved that indeed

$$
S=1 / 2 .
$$

The proof of the lower bound for $S$ by Bohnenblust and Hille is long and involved. A few years later Hartman gave in 20] a different proof for the lower bound, based on probabilistic arguments. From now on $\left(\varepsilon_{n}\right)_{n}$ will always be a sequence of signs in $\{-1,1\}^{\mathbb{N}}$. Let us be more precise. On $\{-1,1\}$ we consider the uniform probability: $\mathbf{p}(-1)=\mathbf{p}(1)=1 / 2$ and, on $\{-1,1\}^{\mathbb{N}}$, the corresponding product probability. We say that $D=\sum a_{n} n^{-s}$ is (uniformly) a.s.-sign convergent on a half plane $[\operatorname{Re}>\sigma]$ whenever $D=\sum \varepsilon_{n} a_{n} n^{-s}$ (uniformly) converges on [Re $>\sigma$ ] outside of a zero set of signs $\varepsilon_{n}$. We will usually average over all possible choices of signs. In order to keep the notation as neat as possible, we will think of $\left(\varepsilon_{n}\right)_{n}$ as a sequence of independent Rademacher random variables on some probability space $\Omega$. We can take of course $\Omega=\{-1,1\}^{\mathbb{N}}$ with the product measure, in which case for each $\omega \in \Omega$, $\varepsilon_{n}(\omega)$ will be the $n$th coordinate of $w$.

\footnotetext{
${ }^{*}$ Departamento de Matematica - Pab I, Facultad de Cs. Exactas y Naturales, Universidad de Buenos Aires, 1428 Buenos Aires (Argentina) and IMAS - CONICET (Argentina). Partially supported by CONICET-PIP 0624, PICT 2011-1456 and UBACyT 20020130100474BA

${ }^{\dagger}$ Institut für Mathematik. Universität Oldenburg. D-26111 Oldenburg (Germany). Supported by MICINN MTM201122417

${ }^{\ddagger}$ Instituto Universitario de Matemática Pura y Aplicada. Universitat Politècnica de València. 46022 Valencia (Spain). Supported by MICINN MTM2011-22417 and UPV-SP20120700
} 
Given a Dirichlet series $D=\sum a_{n} n^{-s}$, Hartman in [20] (with a slightly different notation) considers the following abscissas

$$
\begin{gathered}
\sigma_{c}^{\mathrm{rad}}(D):=\inf \left\{\sigma \in \mathbb{R}: \sum a_{n} n^{-s} \text { a.s.-sign convergent on }[\operatorname{Re}>\sigma]\right\} \\
\sigma_{u}^{\mathrm{rad}}(D):=\inf \left\{\sigma \in \mathbb{R}: \sum a_{n} n^{-s} \text { uniformly a.s.-sign convergent on }[\operatorname{Re}>\sigma]\right\}
\end{gathered}
$$

(obviously, it doesn't make any sense to define an analogous notion like $\sigma_{a}^{\mathrm{rad}}(D)$ ). In general the abscissas $\sigma_{c}^{\mathrm{rad}}(D)$ and $\sigma_{u}^{\mathrm{rad}}(D)$ are different from $\sigma_{c}(D)$ and $\sigma_{u}(D)$, respectively. The two main result in Hartman's article are (compare with (2) and (3))

$$
\sup _{D \text { Dir. ser. }} \sigma_{a}(D)-\sigma_{c}^{\mathrm{rad}}(D)=\frac{1}{2}
$$

and

$$
\sup _{D \text { Dir. ser. }} \sigma_{a}(D)-\sigma_{u}^{\mathrm{rad}}(D)=\frac{1}{2}
$$

Hartman in particular proves that $\sup _{D} \sigma_{a}(D)-\sigma_{u}^{\mathrm{rad}}(D) \leq \sup _{D} \sigma_{a}(D)-\sigma_{u}(D)$, and estimating the first sup from below he produces a substantially different proof of the lower bound of $S$. His proof is probabilistic and goes through almost periodic functions with random Fourier coefficients.

Recently, many authors have shown new interest in the Bohr-Bohnenblust-Hille circle of ideas (see the recent monograph [27] and also [1, 2, 3, 4, 5, 6, 9, 12, 14, 13, 16, 18, 21, 23, 25, 26]), and probabilistic arguments have shown to be of great interest in this theory. Being Hartman's paper the first time when such probabilistic arguments were used to deal with Dirichlet series, our aim in this note is to look at his results from this more modern and more general point of view that we believe clarifies the original argument.

Vector-valued Hardy-type Dirichlet series We are going to work with Hardy spaces of Dirichlet series with values in a Banach space. In this way we continue and extend our work from [9]. Given a Banach space $E$, we consider the one-to one correspondence between the spaces $\mathfrak{P}(E)$ (all formal power series $\sum_{\alpha} c_{\alpha} z^{\alpha}$ in infinitely many variables with coefficients $c_{\alpha} \in E$ ) and $\mathfrak{D}(E)$ (all formal Dirichlet series $\sum_{n} a_{n} \frac{1}{n^{s}}$ with coefficients $a_{n} \in E$ )

$$
\mathfrak{P}(E) \longrightarrow \mathfrak{D}(E), \sum c_{\alpha} z^{\alpha} \mapsto \sum a_{n} n^{-s}
$$

given by $a_{n}=c_{\alpha}$ if $n=p^{\alpha}=p_{1}^{\alpha_{1}} \cdots p_{k}^{\alpha_{k}}$, where $p_{1}<p_{2}<p_{3}<\ldots$ stands for the sequence of prime numbers (see [9]).

Let us recall the definition of Hardy spaces of $E$-valued Dirichlet series (first defined by Bayart for $E=\mathbb{C}$ in $[3]$ and later for arbitrary $E$ in $[9]$ ). For every $f \in L_{1}\left(\mathbb{T}^{\mathbb{N}} ; E\right.$ ) (the Banach space of Bochner integrable $E$-valued functions defined on the infinite dimensional torus $\mathbb{T}^{\mathbb{N}}$ with the normalized Lebesgue measure $d z$ ) and every multi index $\alpha \in \mathbb{Z}^{(\mathbb{N})}$ (all finite sequences $\alpha=\left(\alpha_{n}\right)_{n \in \mathbb{Z}}$ ) we as usual denote the $\alpha$ th Fourier coefficient of $f$ by $\hat{f}(\alpha)=\int_{\mathbb{T}^{\mathbb{N}}} f(z) z^{-\alpha} d z$. Now define for $1 \leq p<\infty$ the Hardy space

$$
H_{p}\left(\mathbb{T}^{\mathbb{N}} ; E\right):=\left\{f \in L_{p}\left(\mathbb{T}^{\mathbb{N}} ; E\right): \hat{f}(\alpha) \neq 0 \text { only if } \alpha \in \mathbb{N}_{0}^{(\mathbb{N})}\right\}
$$

(with the norm induced by $L_{p}\left(\mathbb{T}^{\mathbb{N}} ; E\right)$ ) and let

$$
\mathcal{H}_{p}(E)
$$

by definition be the image of the Banach space $H_{p}\left(\mathbb{T}^{\mathbb{N}} ; E\right)$ by the aforementioned correspondence (6) (again with the norm coming from $H_{p}\left(\mathbb{T}^{\mathbb{N}} ; E\right)$ ). We also consider

$$
\mathcal{H}_{\infty}(E),
$$

the space of $E$-valued Dirichlet series such that $\sum_{n} a_{n} \frac{1}{n^{s}}$ defines a bounded, holomorphic function on $[\operatorname{Re} s>0]$, with the norm $\left\|\sum a_{n} n^{-s}\right\|_{\mathcal{H}_{\infty}(E)}:=\sup _{\operatorname{Re} s>0}\left\|\sum_{n} a_{n} \frac{1}{n^{s}}\right\|_{E}$. We note that this Banach 
space through the identification in (6) coincides isometrically with $H_{\infty}\left(\mathbb{T}^{\mathbb{N}} ; E\right)$ if and only if $E$ has the analytic Radon-Nikodým property (see [17]). In the scalar case we abbreviate

$$
\mathcal{H}_{p}=\mathcal{H}_{p}(\mathbb{C}), 1 \leq p \leq \infty
$$

Clearly, we have that

$$
\mathcal{H}_{2}=\left\{\sum a_{n} n^{-s}:\|D\|_{\mathcal{H}_{2}}=\left(\sum_{n=1}^{\infty}\left|a_{n}\right|^{2}\right)^{\frac{1}{2}}<\infty\right\},
$$

a Hilbert space intensively studied by Hedenmalm, Lindqvist and Seip in [21].

State of art. For any Banach space $E$ and any $1 \leq p \leq \infty$ define the $\mathcal{H}_{p}(E)$-abscissa of a Dirichlet series $\sum a_{n} n^{-s}$ by

$$
\sigma_{\mathcal{H}_{p}(E)}(D):=\inf \left\{\sigma \in \mathbb{R}: \sum \frac{a_{n}}{n^{\sigma}} n^{-s} \in \mathcal{H}_{p}(E)\right\} .
$$

Then, given $1 \leq p \leq \infty$, we have (for $p=\infty$ see [7] (scalar case) and [14, Theorem 1] (vector-valued case), and for $1 \leq p<\infty$ see [2, 3] (scalar case) and [9] (vector-valued case))

$$
S_{p}(E):=\sup _{D \in \mathfrak{D}(E)} \sigma_{a}(D)-\sigma_{\mathcal{H}_{p}(E)}=1-\frac{1}{\cot E} ;
$$

recall that $E$ has cotype $q$ (with $2 \leq q<\infty$ ) if there is a constant $C>0$ such that for every finite choice of elements $x_{1}, \ldots, x_{N} \in E$ we have $\left(\sum_{k}\left\|x_{k}\right\|_{E}^{q}\right)^{\frac{1}{q}} \leq C\left(\int_{\mathbb{T}^{N}}\left\|\sum_{k} x_{k} z_{k}\right\|_{E}^{2} d z\right)^{1 / 2}$, and

$$
\cot E:=\inf \{q \in[2, \infty[: E \text { has cotype } q\} .
$$

Let us comment on the special case $E=\mathbb{C}$ for which $\cot \mathbb{C}=2$. For $p=\infty$ we know by Bohr's fundamental theorem from [8] (see also [27, Theorem 6.2.3]) that

$$
\sigma_{u}(D)=\sigma_{\mathcal{H}_{\infty}}(D),
$$

hence (8) implies (3). For $p=2$ we have

$$
\sigma_{c}^{\mathrm{rad}}(D)=\sigma_{\mathcal{H}_{2}}(D),
$$

so in this case (8) implies (4). Indeed, by Khinchin's inequality it is well-known that a scalar sequence $x=\left(x_{n}\right)$ is a.s.-sign summable (i.e., $\sum_{n} \varepsilon_{n} x_{n}$ converges for almost all possible choices of signs $\left.\varepsilon_{n}\right)$ if and only if $x \in \ell_{2}$. This, together with (7), is what we need. We will come back to this issue later.

Is it also possible to recover (5) within the setting of Hardy-type Dirichlet series? Given $1 \leq p \leq \infty$ and a Banach space $E$, we define what is going to be one of our our main objects,

$$
\mathcal{H}_{p}^{\mathrm{rad}}(E):=\left\{\sum a_{n} n^{-s}: \forall \text { a.e. } \varepsilon_{n}= \pm 1, \sum \varepsilon_{n} a_{n} n^{-s} \in \mathcal{H}_{p}(E)\right\} .
$$

Then, for a given Dirichlet series $D \in \mathfrak{D}(E)$, we consider the abscissa

$$
\sigma_{\mathcal{H}_{p}(E)}^{\mathrm{rad}}(D):=\inf \left\{\sigma \in \mathbb{R}: \sum \frac{a_{n}}{n^{\sigma}} n^{-s} \in \mathcal{H}_{p}^{\mathrm{rad}}(E)\right\},
$$

and again the aim is to determine the maximal distance between $\sigma_{a}(D)$ and $\sigma_{\mathcal{H}_{p}(E)}^{\mathrm{rad}}(D)$.

Summary. Our first main result (Theorem 8) is a proper extension of Hartman's main result from (5) and an analogue of (8) in the setting of a.s.-sign convergence of Hardy-type Dirichlet series: For every Banach space $E$ and $1 \leq p \leq \infty$

$$
S_{p}^{\mathrm{rad}}(E):=\sup _{D \in \mathfrak{D}(E)} \sigma_{a}(D)-\sigma_{\mathcal{H}_{p}(E)}^{\mathrm{rad}}(D)=1-\frac{1}{\cot E} .
$$

Indeed, (12) recovers (5) since $\cot \mathbb{C}=2$ and $\sigma_{\mathcal{H}_{\infty}}^{\mathrm{rad}}(D)$ is the abscissa $\sigma_{u}^{\mathrm{rad}}(D)$ defined by Hartman. Moreover, we show that $S_{p}^{\mathrm{rad}}(E) \leq S_{p}(E)$ (Corollary 7), hence (12) also recovers (8). For the proof of 
(12) we distinguish between finite and infinite dimensional Banach spaces $E$. In the rest of our article we graduate (12). Following an idea from [7], we give precise estimates for the $m$ th graduation of $S_{p}^{\mathrm{rad}}(E)$ along $m$-homogeneous Dirichlet series (see Proposition 9 and Proposition 10). In the scalar case, we graduate (12) along the length of the considered Dirichlet series; here our main results are Theorem 11 and Theorem 12. Finally, in the Appendix we show that the coincidence of the maximum width of the strips of a.s.-sign but not absolute convergence and of uniform a.s.-sign but not absolute convergence coincide (see (4) and (5)) also holds in the vector-valued case.

\section{The Banach space $\mathcal{H}_{p}^{\mathrm{rad}}(E)$}

In this section we collect a few facts on $\mathcal{H}_{p}^{\text {rad }}(E)$ needed later. First of all, we need a norm on $\mathcal{H}_{p}^{\mathrm{rad}}(E)$. We denote by $\left(\varepsilon_{n}\right)_{n}$ the system of Rademacher random variables on $\Omega$. We are going to use the following, fundamental for us, fact (see e.g. [19, Theorem 12.3]): Given a sequence $\left(x_{n}\right)_{n}$ in a Banach space $X$, the series $\sum_{n} \varepsilon_{n} x_{n}$ converges almost surely if and only if $\sum_{n} \varepsilon_{n} x_{n}$ converges in $L_{p}(\Omega ; X)$ for some (and then all) $0<p<\infty$.

Clearly, $\sum_{n} \varepsilon_{n} x_{n}$ converges a.e. is another way to say that $\sum_{n} x_{n}$ is a.s.-sign convergent. Then taking $X=\mathcal{H}_{p}(E)$ and $x_{n}=a_{n} n^{-s} \in \mathcal{H}_{p}(E)$ we can reformulate our space $\mathcal{H}_{p}^{\mathrm{rad}}(E)$ defined in (11) as follows:

$$
\mathcal{H}_{p}^{\mathrm{rad}}(E)=\left\{\sum a_{n} n^{-s}: \sum_{n} \varepsilon_{n} a_{n} n^{-s} \in L_{1}\left(\Omega ; \mathcal{H}_{p}(E)\right)\right\},
$$

and define the norm

$$
\left\|\sum a_{n} n^{-s}\right\|_{\mathcal{H}_{p}^{\mathrm{rad}}(E)}:=\int_{\Omega}\left\|\sum_{n} \varepsilon_{n}(\omega) a_{n} n^{-s}\right\|_{\mathcal{H}_{p}(E)} d \omega
$$

We need $\mathcal{H}_{p}^{\mathrm{rad}}(E)$ to be a Banach space. This follows from the following general result. First, recall (see [19, page 233]) that for a given Banach space $X$, the space $\operatorname{Rad}(X)$ of almost unconditionally summable sequences $\left(x_{n}\right)_{n}$ in $X$ together with the norm

$$
\left\|\left(x_{n}\right)_{n}\right\|_{\operatorname{Rad}(X)}=\int_{\Omega}\left\|\sum_{n} \varepsilon_{n}(\omega) x_{n}\right\|_{X} d \omega
$$

forms a Banach space.

Lemma 1. Let $X$ be a Banach space and let $Y_{n}, n \in \mathbb{N}$ be closed subspaces of $X$. Then $Y=\left\{\left(x_{n}\right)_{n} \in\right.$ $\left.\operatorname{Rad}(X): x_{n} \in Y_{n}\right\}$ is closed in $\operatorname{Rad}(X)$.

Proof. Let us observe first that if $x=\left(x_{n}\right)_{n} \in \operatorname{Rad}(X)$, then $\sum_{m} \varepsilon_{m} x_{m} \in L_{1}(\Omega ; X)$. Due to the orthogonality of the Rademacher system we have

$$
x_{n}=\sum_{m} x_{m} \int_{\Omega} \varepsilon_{m}(\omega) \varepsilon_{n}(\omega) d \omega=\int_{\Omega}\left(\sum_{m} x_{m} \varepsilon_{m}(\omega)\right) \varepsilon_{n}(\omega) d \omega
$$

and this gives

$$
\left\|x_{n}\right\|_{X} \leq \int_{\Omega}\left\|\sum_{m} x_{m} \varepsilon_{m}(\omega)\right\|_{X}\left|\varepsilon_{n}(\omega)\right| d \omega=\int_{\Omega}\left\|\sum_{m} x_{m} \varepsilon_{m}(\omega)\right\|_{X} d \omega=\|x\|_{\operatorname{Rad}(X)} .
$$

Let us take now $\left(x^{(m)}\right)_{m} \in Y$ that converges to a certain $x$ in $\operatorname{Rad}(X)$. We write $x^{(m)}=\left(x_{n}^{(m)}\right)_{n}$ and $x=\left(x_{n}\right)_{n}$, then $\left\|x_{n}^{(m)}-x_{n}\right\|_{X} \leq\left\|x^{(m)}-x\right\|_{\operatorname{Rad}(X)}$, and hence, for each fixed $n$, the sequence $x_{n}^{(m)}$ converges to $x_{n}$ as $m \rightarrow \infty$. Since $x_{n}^{(m)} \in Y_{n}$ for every $n$ and all $Y_{n}$ are closed, we have $x_{n} \in Y_{n}$ for all $n$, or equivalently $x \in Y$.

Proposition 2. For every $1 \leq p \leq \infty$ and every Banach space $E$ the space $\mathcal{H}_{p}^{\mathrm{rad}}(E)$ endowed with the norm defined in (13) is a Banach space. 
Proof. Note that our space $\mathcal{H}_{p}^{\mathrm{rad}}(E)$ is actually a subspace of $\operatorname{Rad}\left(\mathcal{H}_{p}(E)\right)$ :

$$
\mathcal{H}_{p}^{\mathrm{rad}}(E)=\left\{\left(D_{n}\right)_{n} \in \operatorname{Rad}\left(\mathcal{H}_{p}(E)\right): D_{n}=a_{n} n^{-s}, a_{n} \in E\right\} .
$$

Observe that each $F_{n}=\left\{a_{n} n^{-s}: a_{n} \in E\right\} \subseteq \mathcal{H}_{p}(E)$ is isometric to $E$ and hence closed. This by Lemma 1 completes the proof.

The following result is crucial for the modern theory of Dirichlet series: For each $1 \leq p \leq \infty$ there is a constant $C_{p}>1$ such that for any Banach space $E$ and any Dirichlet series $\sum a_{n} n^{-s} \in \mathcal{H}_{p}(E)$ we for every $N$ have

$$
\left\|\sum_{n=1}^{N} a_{n} n^{-s}\right\|_{\mathcal{H}_{p}(E)} \leq C_{p} \log N\left\|\sum_{n=1}^{\infty} a_{n} n^{-s}\right\|_{\mathcal{H}_{p}(E)} .
$$

For $E=\mathbb{C}$ and $p=\infty$ this is a quantification of (9) given in [2, Lemma 1.1] (see also [27, Theorem 6.2.2]), and for $E=\mathbb{C}$ and $p=1$ it is [6, Theorem 3.2]. For $E=\mathbb{C}$ and $1<p<\infty$ the situation is even better, since by [1], the system $\left(n^{-s}\right)_{n \in \mathbb{N}}$ then forms a Schauder basis of $\mathcal{H}_{p}(\mathbb{C})$; hence in this situation the log-term even disappears. The vector-valued case needs an alternative approach - see [15] for a proof which again is very much in the spirit of the starting case $E=\mathbb{C}$ and $p=\infty$ (so of Bohr's original ideas). For our new spaces $\mathcal{H}_{p}^{\mathrm{rad}}(E)$ the situation is much simpler.

Proposition 3. If $1 \leq p \leq \infty, E$ is a Banach space and $\sum a_{n} n^{-s} \in \mathcal{H}_{p}^{\mathrm{rad}}(E)$, then for every $N$ we have

$$
\left\|\sum_{n=1}^{N} a_{n} n^{-s}\right\|_{\mathcal{H}_{p}^{\mathrm{rad}}(E)} \leq\left\|\sum_{n=1}^{\infty} a_{n} n^{-s}\right\|_{\mathcal{H}_{p}^{\mathrm{rad}}(E)} .
$$

Moreover, the sequence of partial sums converges to $\sum_{n=1}^{\infty} a_{n} n^{-s}$ in $\mathcal{H}_{p}^{\mathrm{rad}}(E)$.

Proof. Let us fix $\sum a_{n} n^{-s} \in \mathcal{H}_{p}^{\mathrm{rad}}(E)$ and $N \in \mathbb{N}$. We define $\lambda_{n}=1$ for $1 \leq n \leq N$ and $\lambda_{n}=0$ for $n>N$. We use now the Contraction Principle (see e.g. [19, Theorem 12.2]) to get that for $N<M$,

$$
\begin{aligned}
&\left\|\sum_{n=1}^{N} a_{n} n^{-s}\right\|_{\mathcal{H}_{p}^{\mathrm{rad}}(E)}=\int_{\Omega} \| \sum_{n=1}^{N} \varepsilon_{n}(\omega) a_{n} n^{-s}\left\|_{\mathcal{H}_{p}(E)} d \omega=\int_{\Omega}\right\| \sum_{n=1}^{M} \lambda_{n} \varepsilon_{n}(\omega) a_{n} n^{-s} \|_{\mathcal{H}_{p}(E)} d \omega \\
& \leq \int_{\Omega}\left\|\sum_{n=1}^{M} \varepsilon_{n}(\omega) a_{n} n^{-s}\right\|_{\mathcal{H}_{p}(E)} d \omega=\left\|\sum_{n=1}^{M} a_{n} n^{-s}\right\|_{\mathcal{H}_{p}^{\mathrm{rad}}(E)} .
\end{aligned}
$$

By [19, Theorem 12.3] the series $\sum \varepsilon_{n} a_{n} n^{-s}$ converges in $L_{1}\left(\Omega ; \mathcal{H}_{p}(E)\right)$, hence

$$
\left\|\sum_{n=1}^{M} \varepsilon_{n} a_{n} n^{-s}\right\|_{L_{1}} \longrightarrow\left\|\sum_{n=1}^{\infty} \varepsilon_{n} a_{n} n^{-s}\right\|_{L_{1}} \quad \text { as } \quad M \rightarrow \infty .
$$

By the very definition of the norm in $\mathcal{H}_{p}^{\mathrm{rad}}(E)$ this gives the conclusion.

Our next result shows that for $1 \leq p<\infty$ the study of $\mathcal{H}_{p}^{\mathrm{rad}}(\mathbb{C})$ reduces to the study of $\mathcal{H}_{2}$ (see also Proposition 5 for a vector-valued extension).

Proposition 4. For $1 \leq p<\infty$ we have $\mathcal{H}_{p}^{\mathrm{rad}}(\mathbb{C})=\mathcal{H}_{2}$.

Proof. For fixed $N \in \mathbb{N}$ use the definition of $\mathcal{H}_{p}^{\text {rad }}(\mathbb{C})$, Kahane's inequality (see e.g. [19, Theorem 11.1]), the definition of $\mathcal{H}_{p}(\mathbb{C})$, and finally Khinchin's inequality (see e.g.[19, Theorem 1.10]) in order to get

$$
\begin{aligned}
\left\|\sum_{n=1}^{N} a_{n} n^{-s}\right\|_{\mathcal{H}_{p}^{\mathrm{rad}(\mathbb{C})}} & =\int_{\Omega}\left\|\sum_{n=1}^{N} \varepsilon_{n}(\omega) a_{n} n^{-s}\right\|_{\mathcal{H}_{p}(\mathbb{C})} d \omega \sim\left(\int_{\Omega}\left\|\sum_{n=1}^{N} \varepsilon_{n}(\omega) a_{n} n^{-s}\right\|_{\mathcal{H}_{p}(\mathbb{C})}^{p} d \omega\right)^{\frac{1}{p}} \\
& =\left(\int_{\Omega} \int_{\mathbb{T}^{\mathbb{N}}}\left|\sum_{\alpha} \varepsilon_{p^{\alpha}}(\omega) a_{p^{\alpha}} z^{\alpha}\right|^{p} d z d \omega\right)^{\frac{1}{p}}=\left(\int_{\mathbb{T}^{\mathbb{N}}} \int_{\Omega}\left|\sum_{\alpha} \varepsilon_{p^{\alpha}}(\omega) a_{p^{\alpha}} z^{\alpha}\right|^{p} d \omega d z\right)^{\frac{1}{p}} \\
& \sim\left(\int_{\mathbb{T}^{\mathbb{N}}}\left(\sum_{\alpha}\left|a_{p^{\alpha}} z^{\alpha}\right|^{2}\right)^{\frac{p}{2}} d z\right)^{\frac{1}{p}}=\left(\int_{\mathbb{T}^{\mathbb{N}}}\left(\sum_{\alpha}\left|a_{p^{\alpha}}\right|^{2}\right)^{\frac{p}{2}} d z\right)^{\frac{1}{p}} \\
& =\left(\sum_{\alpha}\left|a_{p^{\alpha}}\right|^{2}\right)^{\frac{1}{2}}=\left(\sum_{n=1}^{N}\left|a_{n}\right|^{2}\right)^{\frac{1}{2}} .
\end{aligned}
$$


This gives the conclusion.

It is not surprising that in the vector-valued situation such a description of $\mathcal{H}_{p}^{\mathrm{rad}}(E)$ is more involved. However, if the space $E$ is nice enough we do have something. Let us recall [24, page 46] that a Banach lattice $E$ is $q$-concave (with $1 \leq q<\infty$ ) if there exists a constant $C>0$ such that for every choice $x_{1}, \ldots, x_{N} \in E$

$$
\left(\sum_{n=1}^{N}\left\|x_{n}\right\|^{q}\right)^{\frac{1}{q}} \leq C\left\|\left(\sum_{n=1}^{N}\left|x_{n}\right|^{q}\right)^{\frac{1}{q}}\right\| .
$$

For a Banach lattice $E$ we define $\widetilde{E\left(\ell_{2}\right)}$ to be the space of sequences $\left(x_{n}\right)_{n=1}^{\infty}$ in $E$ such that

$$
\left\|\left(x_{n}\right)_{n}\right\|_{\overparen{E\left(\ell_{2}\right)}}=\sup _{N}\left\|\left(\sum_{n=1}^{N}\left|x_{n}\right|^{2}\right)^{\frac{1}{2}}\right\|_{E}<\infty .
$$

The closure in $\widetilde{E\left(\ell_{2}\right)}$ of the subspace of finite sequences is denoted by $E\left(\ell_{2}\right)$. We remark that these two spaces coincide if and only if $E$ is weakly sequentially complete (see [24, p. 46] for details).

Proposition 5. If $E$ is a Banach lattice that is $q$ concave for some $q$, then $\mathcal{H}_{p}^{\mathrm{rad}}(E)=E\left(\ell_{2}\right)$ for every $1 \leq p<\infty$.

Proof. Let us first consider $a_{1}, \ldots, a_{N} \in E$. By the very definition of the norms in $\mathcal{H}_{p}^{\mathrm{rad}}(E)$ and $\mathcal{H}_{p}(E)$ and Kahane's inequality (that we apply twice) we have (with constants independent of $N$ )

$$
\begin{aligned}
\left\|\sum_{n=1}^{N} a_{n} n^{-s}\right\|_{\mathcal{H}_{p}^{\mathrm{rad}(E)}}=\int_{\Omega}\left\|\sum_{n=1}^{N} \varepsilon_{n}(\omega) a_{n} n^{-s}\right\|_{\mathcal{H}_{p}(E)} d \omega & \sim\left(\int_{\Omega}\left\|\sum_{n=1}^{N} \varepsilon_{n}(\omega) a_{n} n^{-s}\right\|_{\mathcal{H}_{p}(E)}^{p} d \omega\right)^{\frac{1}{p}} \\
=\left(\int_{\Omega} \int_{\mathbb{T}^{N}}\left\|\sum_{\alpha} \varepsilon_{p^{\alpha}}(\omega) a_{p^{\alpha}} z^{\alpha}\right\|_{E}^{p} d z d \omega\right)^{\frac{1}{p}}= & \left(\int_{\mathbb{T}^{N}} \int_{\Omega}\left\|\sum_{\alpha} \varepsilon_{p^{\alpha}}(\omega) a_{p^{\alpha}} z^{\alpha}\right\|_{E}^{p} d \omega d z\right)^{\frac{1}{p}} \\
& \sim\left(\int_{\mathbb{T}^{N}}\left(\int_{\Omega}\left\|\sum_{\alpha} \varepsilon_{p^{\alpha}}(\omega) a_{p^{\alpha}} z^{\alpha}\right\|_{E} d \omega\right)^{p} d z\right)^{\frac{1}{p}} .
\end{aligned}
$$

But now, since $E$ is $q$ concave for some $q$, for each fixed $z \in \mathbb{T}^{N}$ we have by [24, Theorem 1.d.6]

$$
\int_{\Omega}\left\|\sum_{\alpha} \varepsilon_{p^{\alpha}}(\omega) a_{p^{\alpha}} z^{\alpha}\right\|_{E} d \omega \sim\left\|\left(\sum_{\alpha}\left|a_{p^{\alpha}} z^{\alpha}\right|^{2}\right)^{\frac{1}{2}}\right\|_{E}=\left\|\left(\sum_{n=1}^{N}\left|a_{n}\right|^{2}\right)^{\frac{1}{2}}\right\|_{E} .
$$

This, together with Proposition 3 , yields the conclusion.

\section{A reformulation of $S_{p}^{\mathrm{rad}}(E)$}

Maurizi and Queffélec showed in [25, Theorem 2.4] how $S$ can be characterized in terms of bounds of the norm of the partial sums. A modification of their argument using [15] gives the following vector-valued version: For every $1 \leq p \leq \infty$ and every Banach space $E$

$$
S_{p}(E)=\inf \left\{\sigma>0 \mid \exists c_{\sigma} \forall D=\sum_{n=1}^{N} a_{n} n^{-s} \in \mathcal{H}_{p}(E): \sum_{n=1}^{N}\left\|a_{n}\right\| \leq c_{\sigma} N^{\sigma}\|D\|_{\mathcal{H}_{p}(E)}\right\} .
$$

The original proof of [25, Theorem 2.4] for $E=\mathbb{C}$ uses two key tools. The proof of one inequality is based on a closed-graph argument using the fact that $\mathcal{H}_{p}$ is Banach, and the proof of the converse inequality relies on (14). The results from the preceding section prepare us well to establish the following analogue of (15) within our setting.

Proposition 6. For every $1 \leq p \leq \infty$ and Banach space $E$ we have

$$
S_{p}^{\mathrm{rad}}(E)=\inf \left\{\sigma>0 \mid \exists c_{\sigma} \forall D=\sum_{n=1}^{N} a_{n} n^{-s} \in \mathcal{H}_{p}^{\mathrm{rad}}(E): \sum_{n=1}^{N}\left\|a_{n}\right\| \leq c_{\sigma} N^{\sigma}\|D\|_{\mathcal{H}_{p}^{\mathrm{rad}}(E)}\right\} .
$$


Proof. To show one inequality, let us take $\sigma>S_{p}^{\mathrm{rad}}(E)$. A closed-graph argument (here we need Proposition 22 gives that there exists $c_{\sigma}>0$ such that

$$
\sum_{n=1}^{\infty}\left\|a_{n}\right\| \frac{1}{n^{\sigma}} \leq c_{\sigma}\left\|\sum a_{n} n^{-s}\right\|_{\mathcal{H}_{p}^{\mathrm{rad}}(E)}
$$

for every $\sum a_{n} n^{-s} \in \mathcal{H}_{p}^{\mathrm{rad}}(E)$. Then, for given $a_{1}, \ldots a_{N} \in E$ we have

$$
\sum_{n=1}^{N}\left\|a_{n}\right\| \leq N^{\sigma} \sum_{n=1}^{N} \frac{\left\|a_{n}\right\|}{n^{\sigma}} \leq c_{\sigma} N^{\sigma}\left\|\sum_{n=1}^{N} a_{n} n^{-s}\right\|_{\mathcal{H}_{p}^{\mathrm{rad}}(E)} .
$$

Let us conversely fix now some $\sigma_{0}>0$ satisfying the inequality in Proposition 6 , and choose $\sum a_{n} n^{-s} \in$ $\mathcal{H}_{p}^{\mathrm{rad}}(E)$. By Abel's summation and Proposition 3 we have, for any $\sigma>\sigma_{0}$,

$$
\begin{aligned}
\sum_{n=1}^{N}\left\|a_{n}\right\| \frac{1}{n^{\sigma}}= & \sum_{n=1}^{N}\left\|a_{n}\right\| \frac{1}{N^{\sigma}}+\sum_{n=1}^{N-1} \sum_{k=1}^{n}\left\|a_{k}\right\|\left(\frac{1}{n^{\sigma}}-\frac{1}{(n+1)^{\sigma}}\right) \\
& \leq c_{\sigma_{0}} N^{\sigma_{0}-\sigma}\left\|\sum a_{n} n^{-s}\right\|_{\mathcal{H}_{p}^{\mathrm{rad}}(E)}+\sum_{n=1}^{N-1} c_{\sigma_{0}} n^{\sigma 0}\left\|\sum a_{n} n^{-s}\right\|_{\mathcal{H}_{p}^{\mathrm{rad}}(E)}\left(\frac{1}{n^{\sigma}}-\frac{1}{(n+1)^{\sigma}}\right) .
\end{aligned}
$$

Standard computations following [2, Lemma 1.1] finally give

$$
\sum_{n=1}^{N}\left\|a_{n}\right\| \frac{1}{n^{\sigma}} \leq c_{\sigma_{0}}\left\|\sum a_{n} n^{-s}\right\|_{\mathcal{H}_{p}^{\mathrm{rad}}(E)}\left(1+\sum_{n=1}^{\infty} \frac{\sigma}{n^{\sigma-\sigma_{0}+1}}\right) .
$$

Hence $S_{p}^{\mathrm{rad}}(E) \leq \sigma$ and, since $\sigma$ was arbitrary, the proof is completed.

As an immediate consequence we obtain the following

Corollary 7. For every $1 \leq p \leq \infty$ and Banach space $E$ we have $S_{p}^{\mathrm{rad}}(E) \leq S_{p}(E)$.

Proof. Let us take $\sigma>0$ satisfying the condition in (15). Then for every choice of finitely many $a_{1}, \ldots, a_{N} \in E$ and every $t \in \Omega$ we have

$$
\sum_{n=1}^{N}\left\|a_{n}\right\|=\sum_{n=1}^{N}\left\|\varepsilon_{n}(\omega) a_{n}\right\| \leq c_{\sigma} N^{\sigma}\left\|\sum_{n=1}^{N} \varepsilon_{n}(\omega) a_{n} n^{-s}\right\|_{\mathcal{H}_{p}(E)} .
$$

Integration with respect to $t$ and Proposition 6 give the conclusion.

\section{Uniform a.s.-sign convergence versus absolut covergence for Hardy- type Dirichlet series}

The following theorem is our first main result.

Theorem 8. For every Banach space $E$ and $1 \leq p \leq \infty$ we have

$$
S_{p}^{\operatorname{rad}}(E)=1-\frac{1}{\cot E},
$$

i.e., if a Dirichlet series $\sum a_{n} n^{-s} \in \mathfrak{D}(E)$ is a.e-sign convergent in $\mathcal{H}_{p}(E)$, then $\sum_{n}\left\|a_{n}\right\|_{E} n^{-\sigma}<\infty$ for $\sigma>\sigma_{0}:=1-\frac{1}{\cot E}$, and $\sigma_{0}$ is best possible.

Note that this, in combination with (8), in particular shows that for each Banach space $E$ and each $1 \leq p \leq \infty$ we have

$$
S_{p}^{\mathrm{rad}}(E)=S_{p}(E)
$$

In view of Corollary 7 and (8), we only have to take care of the lower estimate. 
In order to prove Theorem 8, we need the concept of $m$-homogeneous Dirichlet series (that was first suggested in [7] and whose set we denote by $\mathfrak{D}_{m}(E)$ ): Those $\sum a_{n} n^{-s}$ for which $a_{n} \neq 0$ only if $n$ has exactly $m$ prime divisors (counted with multiplicity, we denote this by $\Omega(n)=m$ ). Then we define

$$
\mathcal{H}_{p, m}(E) \text { and } \mathcal{H}_{p, m}^{\mathrm{rad}}(E),
$$

to be the (closed) subspace of $\mathcal{H}_{p}(E)$ and $\mathcal{H}_{p}^{\text {rad }}(E)$, respectively, consisting of $m$-homogeneous Dirichlet series. It is well-known that for all $1 \leq p, q<\infty$ and $m$ (see e.g. [11, Theorem 9.1] or [3])

$$
\mathcal{H}_{p, m}(\mathbb{C})=\mathcal{H}_{q, m}(\mathbb{C}) .
$$

We now can repeat the above program and define for every $m \in \mathbb{N}$, every $1 \leq p \leq \infty$ and every Banach space $E$

$$
\begin{gathered}
S_{p, m}(E):=\sup _{D \in \mathfrak{D}(E) m \text {-hom. }} \sigma_{a}(D)-\sigma_{\mathcal{H}_{p}}(D) \\
S_{p, m}^{\mathrm{rad}}(E):=\sup _{D \in \mathfrak{D}(E) m \text {-hom. }} \sigma_{a}(D)-\sigma_{\mathcal{H}_{p}(E)}^{\mathrm{rad}}(D) ;
\end{gathered}
$$

obviously $S_{p, m}(E) \leq S_{p}(E)$ and $S_{p, m}^{\mathrm{rad}}(E) \leq S_{p}^{\mathrm{rad}}(E)$. Exactly as above (see the proof of Proposition 6 ), we may show that

$$
S_{p, m}(E)=\inf \left\{\sigma>0 \mid \exists c_{\sigma} \forall D=\sum_{n=1}^{N} a_{n} n^{-s} \in \mathcal{H}_{p, m}(E): \sum_{n=1}^{N}\left\|a_{n}\right\| \leq c_{\sigma} N^{\sigma}\|D\|_{\mathcal{H}_{p, m}(E)}\right\}
$$

and

$$
S_{p, m}^{\mathrm{rad}}(E)=\inf \left\{\sigma>0 \mid \exists c_{\sigma} \forall D=\sum_{n=1}^{N} a_{n} n^{-s} \in \mathcal{H}_{p, m}^{\mathrm{rad}}(E): \sum_{n=1}^{N}\left\|a_{n}\right\| \leq c_{\sigma} N^{\sigma}\|D\|_{\mathcal{H}_{p, m}^{\mathrm{rad}}(E)}\right\} .
$$

Moreover, following the argument for Corollary 7 we have

$$
S_{p, m}^{\mathrm{rad}}(E) \leq S_{p, m}(E) .
$$

As a by product of our proof (see the end of Subsection 4.1) we are going to obtain the following result (for the analogue for finite dimensional spaces see Proposition 10):

Proposition 9. For every infinite dimensional Banach space $E$ and every $m$

$$
S_{p, m}^{\mathrm{rad}}(E)=S_{p, m}(E)=1-\frac{1}{\cot E} .
$$

We divide the proof of Theorem 8 into two separate cases: for finite and infinite dimensional spaces.

\subsection{The finite dimensional case}

For every finite dimensional Banach space $E$ we have $\cot E=2$. Then the following counterpart of (9) obviously implies the lower bound in Theorem 8.

Proposition 10. For every finite dimensional Banach space $E$ and every $m$

$$
S_{p, m}^{\mathrm{rad}}(E)=S_{p, m}(E)= \begin{cases}\frac{1}{2} & \text { for } 1 \leq p<\infty \\ \frac{m-1}{2 m} & \text { for } p=\infty\end{cases}
$$

For $p=\infty$ and $E=\mathbb{C}$ this result is due to Bohnenblust-Hille [7] and Hartman [20].

Proof. Since $S_{p, m}^{r a d}(E)$ is invariant under renorming of $E$, we may assume that $E$ is $\mathbb{C}^{k}$ with the euclidean norm. By $(20)$ we need to show the proper lower bound for $S_{p, m}^{\mathrm{rad}}(E)$ and the proper upper bound for $S_{p, m}(E)$. We start with the upper bound for $S_{p, m}(E)$ : Assume first that $1 \leq p<\infty$. Given 
$a_{1}, \ldots, a_{N} \in E$, we then conclude from the Cauchy-Schwarz inequality and the polynomial version of Kahane's inequality [10, Proposition 1.2] that

$$
\begin{aligned}
\sum_{n=1}^{N}\left\|a_{n}\right\| \leq N^{1 / 2}\left(\sum_{n=1}^{N}\left\|a_{n}\right\|^{2}\right)^{1 / 2} & =N^{1 / 2}\left(\int_{\mathbb{T}^{\mathbb{N}}}\left\|\sum_{|\alpha|=m} a_{p^{\alpha}} z^{\alpha}\right\|^{2} d z\right)^{1 / 2} \\
& \sim N^{1 / 2}\left(\int_{\mathbb{T}^{\mathbb{N}}}\left\|\sum_{|\alpha|=m} a_{p^{\alpha}} z^{\alpha}\right\|^{p} d z\right)^{1 / p}=N^{1 / 2}\left\|\sum_{n=1}^{N} a_{n} n^{-s}\right\|_{\mathcal{H}_{p, m}(E)},
\end{aligned}
$$

which by (18) shows what we want. Now for $p=\infty$ we conclude from Hölder's inequality and the polynomial Bohnenblust-Hille inequality (in the form of [16, Theorem 5.3]) that

$$
\begin{aligned}
& \sum_{n=1}^{N}\left\|a_{n}\right\| \leq N^{\frac{2 m}{m-1}}\left(\sum_{n=1}^{N}\left\|a_{n}\right\|^{\frac{2 m}{m+1}}\right)^{\frac{m+1}{2 m}} \\
& \quad \leq C^{m} N^{\frac{2 m}{m-1}} \sup _{z \in \mathbb{T}^{\mathbb{N}}}\left\|\sum_{|\alpha|=m} a_{p^{\alpha}} z^{\alpha}\right\|=C^{m} N^{\frac{2 m}{m-1}}\left\|\sum_{n=1}^{N} a_{n} n^{-s}\right\|_{\mathcal{H}_{\infty, m}(E)},
\end{aligned}
$$

and again (18) gives the conclusion.

Let us turn to the lower bound of $S_{p, m}^{\mathrm{rad}}(E)$ : A simple argument shows that $S_{p, m}^{\mathrm{rad}}(\mathbb{C}) \leq S_{p, m}^{\mathrm{rad}}(E)$, so it remains to estimate $S_{p, m}^{\mathrm{rad}}(\mathbb{C})$ from below. We again start with the case $1 \leq p<\infty$. Then we know from $(17)$ that $S_{p, m}^{\mathrm{rad}}(\mathbb{C})=S_{2, m}^{\mathrm{rad}}(\mathbb{C})$, and hence we may concentrate on the case $p=2$.

Clearly $S_{2, m}^{\mathrm{rad}}(\mathbb{C}) \geq S_{2,1}^{\mathrm{rad}}(\mathbb{C})$, then we can assume that $\sigma>0$ and $c_{\sigma}>0$ are as in (18) with $p=2$, $m=1$ and $E=\mathbb{C}$. Hence by the prime number theorem there are constants $C_{1}, C_{2}>0$ such that

$$
\frac{N}{\log N} \leq C_{1} \sum_{\substack{n=1 \\ \Omega(n)=1}}^{N} 1 \leq c_{\sigma} N^{\sigma}\left\|\sum_{\substack{n=1 \\ \Omega(n)=1}}^{N} n^{-s}\right\|_{\mathcal{H}_{2, m}^{\mathrm{rad}}(\mathbb{C})} \leq C_{2} c_{\sigma} N^{\sigma}\left(\frac{N}{\log N}\right)^{1 / 2},
$$

and this is exactly what we need.

Finally, we consider the case $p=\infty$ : We fix $\sigma>S_{\infty, m}^{\mathrm{rad}}(\mathbb{C})$; by a standard closed graph argument there is a constant $c_{\sigma}>0$ such that

$$
\sum_{n=1}^{\infty}\left|a_{n}\right| \frac{1}{n^{\sigma}} \leq c_{\sigma}\left\|\sum_{n} a_{n} n^{-s}\right\|_{\mathcal{H}_{\infty}^{\mathrm{rad}(\mathbb{C})}} .
$$

We consider $\varepsilon_{\alpha}$ independent Rademacher random variables (i.e. each one taking values \pm 1 with probability $1 / 2$ ) for $\alpha \in \mathbb{N}_{0}^{N}$ with $|\alpha|=m$. By the Kahane-Salem-Zygmund inequality, as presented in [27, Theorem 5.3.4] there is a constant $C>0$ such that

$$
\int \sup _{z \in \mathbb{D}^{N}}\left|\sum_{\substack{\alpha \in \mathbb{N}_{0}^{N} \\|\alpha|=m}} \varepsilon_{\alpha}(\omega) z^{\alpha}\right| d \omega \leq C\left(\sum_{\substack{\alpha \in \mathbb{N}_{0}^{N} \\|\alpha|=m}} 1\right)^{\frac{1}{2}} \sqrt{N \log m} \leq C N^{\frac{m+1}{2}} \sqrt{\log m} .
$$

We consider now the polynomial $\sum_{\alpha \in \mathbb{N}_{0}^{N}} z^{\alpha}$ and denote by $D_{N}$ the Dirichlet series associated to it by (6). Then

$$
\left\|D_{N}\right\|_{\mathcal{H}_{\infty}^{\mathrm{rad}}(\mathbb{C})}=\int_{\Omega} \sup _{z \in \mathbb{D}^{N}}\left\|\sum_{\substack{\alpha \in \mathbb{N}_{0}^{N} \\|\alpha|=m}} r_{p^{\alpha}}(t) z^{\alpha}\right\| d \omega \leq C N^{\frac{m+1}{2}} \sqrt{\log m} .
$$

With this and $(22)$ we get that, for every $N$

$$
\sum_{\substack{\alpha \in \mathbb{N}_{0}^{N} \\|\alpha|=m}} \frac{1}{p^{\alpha \sigma}} \leq c_{\sigma} C N^{\frac{m+1}{2}} \sqrt{\log m}
$$


All we need now is a lower bound of $\sum_{|\alpha|=m} \frac{1}{p^{\alpha \sigma}}$. By a weak consequence of the Prime Number Theorem $p_{j} \sim j \log j$. Then for a fixed $\varepsilon>0$ there is a constant $B>0$ such that for all $j$ we have $p_{j} \leq B j^{1+\varepsilon}$, hence

$$
\sum_{|\alpha|=m} \frac{1}{p^{\alpha \sigma}}=\sum_{1 \leq j_{1} \leq \ldots \leq j_{m} \leq N} \frac{1}{\left(p_{j_{1}} \cdots p_{j_{m}}\right)^{\sigma}} \geq \frac{1}{B^{m}} \sum_{1 \leq j_{1} \leq \ldots \leq j_{m} \leq N} \frac{1}{\left(j_{1} \ldots j_{N}\right)^{(1+\varepsilon) \sigma}} .
$$

Let us now observe that

$$
\sum_{j_{1}, \ldots, j_{m}=1}^{N} \frac{1}{\left(j_{1} \cdots j_{m}\right)^{(1+\varepsilon) \sigma}} \leq \sum_{1 \leq j_{1} \leq \ldots \leq j_{m} \leq N} m ! \frac{1}{\left(j_{1} \cdots j_{m}\right)^{(1+\varepsilon) \sigma}} .
$$

Then

$$
\begin{aligned}
\sum_{1 \leq j_{1} \leq \ldots \leq j_{m} \leq N} \frac{1}{\left(j_{1} \ldots j_{m}\right)^{(1+\varepsilon) \sigma}} & \geq \frac{1}{m !} \sum_{j_{1}, \ldots, j_{N}=1}^{N} \frac{1}{\left(j_{1} \ldots j_{m}\right)^{(1+\varepsilon) \sigma}} \\
& =\frac{1}{m !}\left(\sum_{j=1}^{N} \frac{1}{j^{(1+\varepsilon) \sigma}}\right)^{m} \geq D \frac{N^{m}}{N^{(1+\varepsilon) \sigma m}} .
\end{aligned}
$$

This altogether gives that there is a constant $K_{m}$ depending only on $m$ such that

$$
N^{m(1-(1+\varepsilon) \sigma)} \leq K_{m} \sqrt{\log m} N^{\frac{m+1}{2}},
$$

which yields $\frac{m-1}{2 m} \leq \sigma$ and gives the result.

\subsection{The infinite dimensional case}

Let us now prove Theorem 8 for infinite dimensional Banach spaces $E$. Once again, by Corollary 7 and equation (8), it suffices to check the following: Given an infinite dimensional Banach space $E$ and $1 \leq p \leq \infty$ the following holds

$$
1-\frac{1}{\cot E} \leq S_{p}^{\mathrm{rad}}(E)
$$

Proof. For each fixed $t \in \Omega$ we have

$$
\left\|\sum \varepsilon_{n}(\omega) a_{n} n^{-s}\right\|_{\mathcal{H}_{p}(E)} \leq\left\|\sum \varepsilon_{n}(\omega) a_{n} n^{-s}\right\|_{\mathcal{H}_{\infty}(E)} .
$$

Integrating with respect to $t$ we get that $\mathcal{H}_{\infty}^{\mathrm{rad}}(E) \subset \mathcal{H}_{p}^{\mathrm{rad}}(E)$ for every $1 \leq p \leq \infty$. Hence to find a lower bound for $S_{p}^{\mathrm{rad}}(E)$ it is enough to get some lower estimate for $S_{\infty}^{\mathrm{rad}}(E)$. What we are going to do is to work only with 1-homogeneous Dirichlet series, finding lower bounds for $S_{\infty, 1}^{\mathrm{rad}}(E)$. Recall from (18) that

$$
S_{\infty, 1}^{\mathrm{rad}}(E)=\inf \left\{\sigma>0: \exists c_{\sigma} \forall a_{p_{1}}, \ldots, a_{p_{N}} \in E: \sum_{k=1}^{N}\left\|a_{p_{k}}\right\| \leq c_{\sigma} p_{N}^{\sigma}\left\|\sum_{k=1}^{N} a_{p_{k}} p_{k}^{-s}\right\|_{\mathcal{H}_{\infty}^{\mathrm{rad}(E)}}\right\} .
$$

On the other hand for each $\omega$,

$$
\left\|\sum_{k=1}^{N} \varepsilon_{p_{k}}(\omega) a_{p_{k}} p_{k}^{-s}\right\|_{\mathcal{H}_{\infty}(E)}=\sup _{u \in \mathbb{T}^{N}}\left\|\sum_{k=1}^{N} \varepsilon_{p_{k}}(\omega) a_{p_{k}} u_{k}\right\|_{E}=\sup _{w \in \mathbb{T}^{N}}\left\|\sum_{k=1}^{N} a_{p_{k}} w_{k}\right\|_{E}=\left\|\sum_{k=1}^{N} a_{p_{k}} p_{k}^{-s}\right\|_{\mathcal{H}_{\infty}(E)} .
$$

Now, integrating on $t$ we obtain

$$
\left\|\sum_{k=1}^{N} a_{p_{k}} p_{k}^{-s}\right\|_{\mathcal{H}_{\infty}^{\mathrm{rad}(E)}}=\int_{\Omega}\left\|\sum_{k=1}^{N} \varepsilon_{p_{k}}(\omega) a_{p_{k}} p_{k}^{-s}\right\|_{\mathcal{H}_{\infty}(E)} d \omega=\left\|\sum_{k=1}^{N} a_{p_{k}} p_{k}^{-s}\right\|_{\mathcal{H}_{\infty}(E)} .
$$

This means that $S_{\infty, 1}^{\mathrm{rad}}(E)=S_{\infty, 1}(E)$. But from [14, p.554] we know that $S_{\infty, 1}(E)=1-\frac{1}{\cot E}$ which completes the proof.

A brief analysis of the preceding proof shows that we also get Proposition 9 as a by-product. 


\section{$5 \quad$ Sharp estimates}

By definition the $x$ th Sidon constant for Dirichlet series is given by

$$
S_{\infty}(x):=\sup _{\left(a_{n}\right)_{n \in \mathbb{N}} \subseteq \mathbb{C}} \frac{\sum_{n \leq x}\left|a_{n}\right|}{\left\|\sum_{n \leq x} a_{n} n^{-s}\right\|_{\mathcal{H}_{\infty}(\mathbb{C})}},
$$

and its (almost) precise asymptotic is expressed in the following formula:

$$
S_{\infty}(x)=\frac{\sqrt{x}}{e^{\left(\frac{1}{\sqrt{2}}+o(1)\right) \sqrt{\log x \log \log x}}}
$$

this results with weaker constants instead of $\frac{1}{\sqrt{2}}$ was proved in [23, Theorem 4.3], the lower estimate was given in [12, Théorème 1.1], and finally the upper estimate followed from the hypercontractivity of the Bohnenblust-Hille inequality in [13, Theorem 1]. In view of the characterization (15), equation (25) represents a sharp estimate of the largest possible width on which a Dirichlet series $D=\sum a_{n} n^{-s}$ converges uniformly but not absolutely. Given $x \geq 2$ and $1 \leq p \leq \infty$, asymptotically correct estimates for

$$
S_{p}(x):=\sup _{\left(a_{n}\right)_{n \in \mathbb{N}} \subseteq \mathbb{C}} \frac{\sum_{n \leq x}\left|a_{k}\right|}{\left\|\sum_{n \leq x} a_{n} \frac{1}{n^{s}}\right\|_{\mathcal{H}_{p}(\mathbb{C})}}
$$

like (25) are unfortunately so far unknown for $p \neq 2$. For $p=2$ we have $S_{2}(x)=\sqrt{x}$ by (7). An analogue of this definition in our probabilistic setting à la Hartman is (again $x \in \mathbb{N}$ and $1 \leq p \leq \infty$ )

$$
S_{p}^{\mathrm{rad}}(x):=\sup _{\left(a_{n}\right)_{n \in \mathbb{N}} \subseteq \mathbb{C}} \frac{\sum_{n \leq x}\left|a_{n}\right|}{\left\|\sum_{n \leq x} a_{n} \frac{1}{n^{s}}\right\|_{\mathcal{H}_{p}^{\mathrm{rad}}(\mathbb{C})}} .
$$

Proposition 6 and Theorem 8 (for $E=\mathbb{C}$ ) suggest the following analogue of 25 ). It can be seen as the definite result of Hartman's original question.

Theorem 11. We have, as $x$ tends to $\infty$

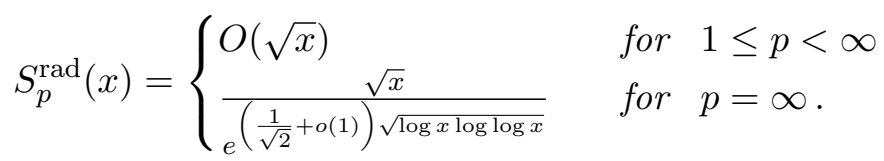

The formula for $1 \leq p<\infty$ is an immediate consequence of Proposition 4. Let us deal with the case $p=\infty$. We first prove that for every $x$ and $p$

$$
S_{p}^{\operatorname{rad}}(x) \leq S_{p}(x)
$$

then the upper estimate for $S_{\infty}^{\mathrm{rad}}(x)$ obviously follows from (25). By definition $S_{p}^{\mathrm{rad}}(x)$ is the best constant $C>0$ such that for all sequences $\left(a_{n}\right)_{n \in \mathbb{N}} \subseteq \mathbb{C}$ we have $\sum_{n \leq x}\left|a_{n}\right| \leq C\left\|\sum_{n \leq x} a_{n} n^{-s}\right\|_{\mathcal{H}_{p}(\mathbb{C})}$. But for each $\omega \in \Omega$

$$
\sum_{n \leq x}\left|a_{n}\right|=\sum_{n \leq x}\left|a_{n} \varepsilon_{n}(\omega)\right| \leq C\left\|\sum_{n \leq x} \varepsilon_{n}(\omega) a_{n} n^{-s}\right\|_{\mathcal{H}_{p}(\mathbb{C})},
$$

so that (27) follows by integration. It remains to prove the lower estimate for $S_{\infty}^{\mathrm{rad}}(x)$ in Theorem 11 , and the arguments we give follow from an analysis of the proof for (25). Our presentation is close to that of [12] and also [27, Theorem 5.4.3], and it is mainly given for the sake of completeness. Before we start we need some preparation from analytic number theory.

Given $k \in \mathbb{N}$, define $\mathcal{J}(k)=\left\{\mathbf{j}=\left(j_{1}, \ldots, j_{k}\right) \in \mathbb{N}_{0}^{k}: 1 \leq j_{1} \leq \cdots \leq j_{k}\right\}$, and for any sequence $z=\left(z_{n}\right)$ of complex numbers and any $\mathbf{j} \in \mathcal{J}(k)$ let $z_{\mathbf{j}}=z_{j_{1}} \cdots z_{j_{k}}$. Moreover, for $x>2$ and $2<y \leq x$, choose $\ell \in \mathbb{N}$ such that $p_{\ell} \leq y<p_{\ell+1}$ (note that with the usual notation from number theory $\ell=\pi(y)$ ). With $x$ and $\ell$ define the index set

$$
J^{-}(x ; y)=\left\{\mathbf{j}=\left(j_{1}, \ldots, j_{k}\right) \in \mathcal{J}(k): k \in \mathbb{N}, p_{\mathbf{j}} \leq x, j_{k} \leq \ell\right\} .
$$


Note first that $2^{\text {length }(\mathbf{j})} \leq p_{\mathbf{j}} \leq x$ for every $\mathbf{j} \in J^{-}(x ; y)$, hence the maximal length

$$
L:=\max \left\{\operatorname{length}(\mathbf{j}): \mathbf{j} \in J^{-}(x ; y)\right\} \leq \frac{\log x}{\log 2} .
$$

The asymptotic behavior of the function $\left|J^{-}(x ; y)\right|$ is very well described by the so called Dickmann function $\varrho:[0, \infty[\rightarrow \mathbb{R}$ which is uniquely determined through the following conditions:

- $\varrho$ is differentiable on $] 1, \infty[$ where it satisfies the differential equation

$$
u \varrho^{\prime}(u)+\varrho(u-1)=0 .
$$

- $\varrho(u)=1$ for all $0 \leq u \leq 1$, and $\varrho$ is continuous at 1 .

For this definition see e.g. 28, III.5, p. 365,370]. We need the following two asymptotic estimates; the first one can be found in [28, III.5.5, Corollary 9.3] (see also [22, Eq. (1.8)]), and the second in [22, Eq. (1.7)]:

- Given $\varepsilon>0$, there is $C=C(\varepsilon)>0$ such for all $x, y$ with $x>2$ and $e^{(\log \log x)^{\frac{5}{3}+\varepsilon}} \leq y \leq x$

$$
\frac{1}{C} x \varrho(u) \leq\left|J^{-}(x ; y)\right| \leq C x \varrho(u),
$$

where here (and in the sequel) $u=\frac{\log x}{\log y}$.

- For $u \rightarrow \infty$ :

$$
\log \varrho(u)=-u \log u(1+o(1))
$$

We are now ready to start the

Proof of the lower estimate of $S^{\mathrm{rad}}(x)$ in Theorem 11. Fix $x>2$, and choose some $2<y \leq x$ together with some $\ell \in \mathbb{N}$ for which $p_{\ell} \leq y<p_{\ell+1}$ (later it will turn out that the optimal choice for $y$ in fact is $y=e^{\frac{1}{\sqrt{2}} \sqrt{\log x \log \log x}}$. The general strategy will be to apply in a first step the Kahane-Salem-Zygmund inequality [27, Theorem 5.3.4] in order to get

$$
\sqrt{\frac{\left|J^{-}(x ; y)\right|}{y \log \log x}} \leq K S^{\mathrm{rad}}(x)
$$

for some universal $K$ and then in a second step to optimize $y$ with analytic number theory.

Define the finite Dirichlet series

$$
D_{x, y}=\sum_{\mathbf{j} \in J^{-}(x ; y)} \frac{1}{p_{\mathbf{j}}^{s}},
$$

which obviously has length $\leq x$. Clearly

$$
\sum_{\mathbf{j} \in J^{-}(x ; y)} 1=\left|J^{-}(x ; y)\right|
$$

and therefore our aim for the proof of (31) will be to show

$$
\left\|D_{x, y}\right\|_{\mathcal{H}_{\infty}^{\mathrm{rad}}} \leq K \sqrt{y\left|J^{-}(x ; y)\right| \log \log x} .
$$

By Bohr's fundamental lemma (see e.g. [27, Theorem 4.4.2]) we have

$$
\left\|D_{x, y}\right\|_{\mathcal{H}_{\infty}^{\text {rad }}}=\int_{0}^{1} \sup _{z \in \mathbb{T}^{\ell}}\left|\sum_{\mathbf{j} \in J^{-}(x ; y)} r_{p_{\mathbf{j}}}(t) z_{\mathbf{j}}\right| d \omega .
$$

Hence by (28) we deduce from the Kahane-Salem-Zygmund inequality (see the version given in [27, Theorem 5.3.4]) that

$$
\left\|D_{x, y}\right\|_{\mathcal{H}_{\infty}^{\mathrm{rad}}} \leq K \sqrt{\ell\left|J^{-}(x ; y)\right| \log \log x} .
$$


But trivially $\ell \leq y$ which gives (32) and hence (31). To finish the proof the number theoretical results from $(29)$ and $(30)$ enter the game. Assume that $y=e^{\alpha \sqrt{\log x \log \log x}}$, where $\alpha>0$ will be specified later (as already noted it will turn out that the perfect choice is $\alpha=\frac{1}{\sqrt{2}}$ ). Put

$$
u:=\frac{\log x}{\log y}=\frac{1}{\alpha} \sqrt{\frac{\log x}{\log \log x}} .
$$

A simple calculation then gives

$$
u \log u=\frac{1}{2 \alpha} \sqrt{\log x \log \log x}(1+o(1)) .
$$

Note also that, taking for example $\varepsilon=1, y$ lies in the interval of validity of inequality (29). Then we have:

$$
\begin{gathered}
S^{\mathrm{rad}}(x) \stackrel{\sqrt[31]{31}, \sqrt{29})}{\geq} K_{1} \sqrt{\frac{x}{\log \log x}} \sqrt{\frac{\varrho(u)}{y}} \\
\stackrel{\text { def. of } y}{=} K_{1} \sqrt{\frac{x}{\log \log x}} e^{\frac{\log \varrho(u)}{2}} e^{-\frac{\alpha}{2} \sqrt{\log x \log \log x}} \\
\stackrel{300, \sqrt[331]{\geq}}{=} K_{2} \sqrt{\frac{x}{\log \log x}} e^{-\left(\frac{1}{4 \alpha}+\frac{\alpha}{2}+o(1)\right) \sqrt{\log x \log \log x}}=K_{2} \sqrt{x} e^{-\left(\frac{1}{4 \alpha}+\frac{\alpha}{2}+o(1)\right) \sqrt{\log x \log \log x}}
\end{gathered}
$$

Minimizing $\frac{1}{4 \alpha}+\frac{\alpha}{2}$ yields the optimal parameter $\alpha=\frac{1}{\sqrt{2}}$, and we finally arrive at the desired lower estimate for $S^{\mathrm{rad}}(x)$ in Theorem 11 .

Again it is possible to graduate the result from Theorem 11 along $m$-homogeneous polynomials. As in (24) and (26) we may define

$$
S_{p, m}(x) \text { and } S_{p, m}^{\mathrm{rad}}(x), x \in \mathbb{N}
$$

replacing $\mathcal{H}_{p}$ by $\mathcal{H}_{p, m}$ as well as $\mathcal{H}_{p}^{\mathrm{rad}}$ by $\mathcal{H}_{p, m}^{\mathrm{rad}}$, and again we see that $S_{p, m}^{\mathrm{rad}}(x) \leq S_{p, m}(x)$. A careful analysis of [2, Theorem 1.4] and [25, Theorem 3.1] (see also [18]) proves

$$
S_{\infty, m}(x)=O\left(\frac{x^{\frac{m-1}{2 m}}}{(\log x)^{m-1}}\right),
$$

and then the following $m$-homogeneous variant of Theorem 11 comes naturally.

Theorem 12. We have, as $x$ tends to $\infty$

$$
S_{p, m}^{\mathrm{rad}}(x)= \begin{cases}O(\sqrt{x}) & \text { for } 1 \leq p<\infty \\ O\left(\frac{x^{\frac{m-1}{2 m}}}{(\log x)^{m-1}}\right) & \text { for } p=\infty .\end{cases}
$$

Only the lower estimates have to be checked. For the case $1 \leq p<\infty$ argue as in the proof of Theorem 10. For $p=\infty$ analyse again the proof of the lower estimate in (34).

\section{Appendix: On the abscissa of a.s.-sign convergence}

One of the remarkable results of the work of Hartman [20] was that, unlike the classical strips ((2) and (3)), the maximal width of the two strips of the a.s.-sign convergence coincide (44) and (5)). We already pointed out 10 that this result fits in our point of view and in fact follows from our Theorem 8 ,

We wonder now what happens with the abscissas of a.s.-sign convergence and absolute convergence for vector-valued Dirichlet series. Will it again be the case that the maximal distance between these two is the same as the maximal width for the abscissa of a.s.-sign uniform and absolute convergence? We 
answer this question now. Let us introduce some notation just for this appendix; for a given Banach space $E$ we consider the numbers

$$
\begin{aligned}
S_{c \rightarrow a}^{\mathrm{rad}}(E) & :=\sup _{D \in \mathfrak{D}(E)} \sigma_{a}(D)-\sigma_{c}^{\mathrm{rad}}(D) \\
S_{u \rightarrow a}^{\mathrm{rad}}(E) & :=\sup _{D \in \mathfrak{D}(E)} \sigma_{a}(D)-\sigma_{u}^{\mathrm{rad}}(D)
\end{aligned}
$$

By $S_{m, c \rightarrow a}^{\mathrm{rad}}(E)$ and $S_{m, u \rightarrow a}^{\mathrm{rad}}(E)$ we denote their graduations along the homogeneity $m \in \mathbb{N}$, defined in the obvious way. Observe that $S_{u \rightarrow a}^{\mathrm{rad}}(E)$ and $S_{m, u \rightarrow a}^{\mathrm{rad}}(E)$ are just the $S_{\infty}^{\mathrm{rad}}(E)$ and $S_{\infty, m}^{\mathrm{rad}}(E)$ that we considered before.

Obviously we have the trivial estimates

$$
S_{m, u \rightarrow a}^{\mathrm{rad}}(X) \leq S_{u \rightarrow a}^{\mathrm{rad}}(X) \text { and } S_{m, c \rightarrow a}^{\mathrm{rad}}(X) \leq S_{c \rightarrow a}^{\mathrm{rad}}(X)
$$

as well as

$$
S_{u \rightarrow a}^{\mathrm{rad}}(X) \leq S_{c \rightarrow a}^{\mathrm{rad}}(X) \text { and } S_{m, u \rightarrow a}^{\mathrm{rad}}(X) \leq S_{m, c \rightarrow a}^{\mathrm{rad}}(X) .
$$

Our aim is to show that for every Banach space $E$ we have

$$
S_{u \rightarrow a}^{\mathrm{rad}}(E)=S_{c \rightarrow a}^{\mathrm{rad}}(E)=S_{m, c \rightarrow a}^{\mathrm{rad}}(E)=1-\frac{1}{\cot (E)} ;
$$

and if $E$ is infinite-dimensional, then we can also put $S_{m, u \rightarrow a}^{\mathrm{rad}}(E)$ within the previous inequalities. The equalities for $S_{u \rightarrow a}^{\mathrm{rad}}(E)$ and $S_{m, u \rightarrow a}^{\mathrm{rad}}(E)$ follow from Theorem 8 and Proposition 9 with $p=\infty$. We once again mention that the scalar case $E=\mathbb{C}$ is due to Bohr, Bohnenblust-Hille and Hartman.

We consider again the space

$$
\operatorname{Rad}(E):=\left\{a=\left(a_{n}\right) \in E^{\mathbb{N}}: \sum_{n=1}^{\infty} a_{n} \varepsilon_{n} \in L_{1}(\Omega ; E)\right\}
$$

which together with the norm

$$
\left\|\left(a_{n}\right)_{n}\right\|_{\operatorname{Rad}(E)}:=\int_{0}^{1}\left\|\sum_{n=1}^{\infty} a_{n} \varepsilon_{n}(\omega)\right\|_{E} d \omega
$$

forms a Banach space. Recall that $\left(a_{n}\right)_{n}$ belongs to $\operatorname{Rad}(E)$ if and only if $\sum_{n=1}^{\infty} a_{n} \varepsilon_{n}$ converges for almost all choices of signs $\varepsilon_{n}$. In particular,

$$
\sigma_{c}^{\operatorname{rad}}(D)=\inf \left\{\sigma \in \mathbb{R}:\left(\frac{a_{n}}{n^{\sigma}}\right) \in \operatorname{Rad}(E)\right\} .
$$

Let us note that the key ingredient to get descriptions of the width of the strip in the spirit of Maurizi-Queffélec (see (15), (16), (18) and (19)) is to have a norm that provides a proper control of the size of the partial sums, like in (14). Observe that now, by Kahane's contraction principle, we have that for each $N$

$$
\left\|\left(a_{n}\right)_{n=1}^{N}\right\|_{\operatorname{Rad}(E)} \leq\left\|\left(a_{n}\right)\right\|_{\operatorname{Rad}(E)} .
$$

Proceeding as in Proposition 6, using this instead of Proposition 3, we obtain

$$
\begin{aligned}
S_{c \rightarrow a}^{\mathrm{rad}}(E) & =\inf \left\{\sigma>0 \mid \exists c_{\sigma} \forall D=\sum_{n=1}^{N} a_{n} n^{-s} \in \mathfrak{D}(E): \sum_{n=1}^{N}\left\|a_{n}\right\| \leq c_{\sigma} N^{\sigma}\left\|\left(a_{n}\right)_{n=1}^{N}\right\|_{\operatorname{Rad}(E)}\right\} \\
S_{m, c \rightarrow a}^{\mathrm{rad}}(E) & =\inf \left\{\sigma>0 \mid \exists c_{\sigma} \forall D=\sum_{n=1}^{N} a_{n} n^{-s} \in \mathfrak{D}_{m}(E): \sum_{n=1}^{N}\left\|a_{n}\right\| \leq c_{\sigma} N^{\sigma}\left\|\left(a_{n}\right)_{n=1}^{N}\right\|_{\operatorname{Rad}(E)}\right\}
\end{aligned}
$$

Note that in the scalar case $E=\mathbb{C}$, by Khinchin's inequality, we see that

$$
S_{c \rightarrow a}^{\mathrm{rad}}(\mathbb{C})=\inf \left\{\sigma>0\left|\exists c_{\sigma} \forall D=\sum_{n=1}^{N} a_{n} n^{-s} \in \mathfrak{D}(\mathbb{C}): \sum_{n=1}^{N}\right| a_{n} \mid \leq c_{\sigma} N^{\sigma}\left(\sum_{n=1}^{N}\left|a_{n}\right|^{2}\right)^{\frac{1}{2}}\right\},
$$

and hence, applying the Cauchy-Schwarz inequality, we obtain Hartman's result $S_{c \rightarrow a}^{\mathrm{rad}}(\mathbb{C})=\frac{1}{2}$. Finally to complete the proof of (37) it only remains to show the following 
Theorem 13. For every Banach space $E$ and every $m \in \mathbb{N}$

$$
S_{c \rightarrow a}^{\mathrm{rad}}(E)=S_{m, c \rightarrow a}^{\mathrm{rad}}(E)=1-\frac{1}{\cot (E)} .
$$

Proof. We begin with the equality for $S_{c \rightarrow a}^{\mathrm{rad}}(E)$. By (35) and the lower estimate for $S_{u \rightarrow a}^{\mathrm{rad}}(E)$ from (37) we have to check

$$
S_{c \rightarrow a}^{\mathrm{rad}}(E) \leq 1-\frac{1}{\cot (E)} .
$$

Take $q>\cot (E)$. Then for each finite Dirichlet series $D=\sum_{n=1}^{N} a_{n} \frac{1}{n^{s}} \in \mathfrak{D}(E)$ by Hölder's inequality

$$
\sum_{n=1}^{N}\left\|a_{n}\right\| \leq N^{\frac{1}{q^{\prime}}}\left(\sum_{n=1}^{N}\left\|a_{n}\right\|^{q}\right)^{\frac{1}{q}} \leq C_{q}(E) N^{\frac{1}{q^{\prime}}}\left\|\left(a_{n}\right)\right\|_{\operatorname{Rad}(E)} .
$$

Hence we obtain from (39) that $S_{c \rightarrow a}^{\mathrm{rad}} \leq 1-\frac{1}{q}$, the conclusion.

We finish by giving the argument for $S_{m, c \rightarrow a}^{\mathrm{rad}}(E)$. If $E$ is infinite-dimensional, by (35) and (36), the result for $S_{m, u \rightarrow a}^{\mathrm{rad}}(E)$ from (37), and (41) we have

$$
1-\frac{1}{\cot (E)}=S_{m, u \rightarrow a}^{\mathrm{rad}}(E) \leq S_{m, c \rightarrow a}^{\mathrm{rad}}(E) \leq S_{c \rightarrow a}^{\mathrm{rad}}(E)=1-\frac{1}{\cot (E)} .
$$

On the other hand, if $E$ is finite dimensional we can argue as in 21 to show that $S_{1, c \rightarrow a}^{\mathrm{rad}}(\mathbb{C}) \geq 1 / 2$. This completes the proof.

In the scalar case $E=\mathbb{C}$ and in view of Proposition 38, it is again possible to graduate $S_{c \rightarrow a}^{\mathrm{rad}}(\mathbb{C})$ and $S_{m, c \rightarrow a}^{\mathrm{rad}}(\mathbb{C})$, respectively, along the length of the Dirichlet polynomials. As in $(24)$, for $x \geq 1$ we define

$$
S_{c \rightarrow a}^{\mathrm{rad}}(x):=\sup _{\left(a_{n}\right)_{n \in \mathbb{N} \subseteq \mathbb{C}}} \frac{\sum_{n \leq x} a_{n} \mid}{\left\|\left(a_{n}\right)_{n \leq x}\right\|_{\operatorname{Rad}(E)}},
$$

and similarly $S_{m, c \rightarrow a}^{\mathrm{rad}}(x)$. Then by Khinchin's inequality and the Cauchy-Schwarz inequality we obviously have

$$
S_{c \rightarrow a}^{\mathrm{rad}}(x)=S_{m, c \rightarrow a}^{\mathrm{rad}}(x) \sim \sqrt{x} .
$$

\section{Acknowledgement}

The authors wish to thank the referee for her/his valuable remarks.

\section{References}

[1] A. Aleman, J.-F. Olsen, and E. Saksman. Fourier multipliers for Hardy spaces of Dirichlet series. Int. Math. Res. Not. IMRN, 16:4368-4378, 2014.

[2] R. Balasubramanian, B. Calado, and H. Queffélec. The Bohr inequality for ordinary Dirichlet series. Studia Math., 175(3):285-304, 2006.

[3] F. Bayart. Hardy spaces of Dirichlet series and their composition operators. Monatsh. Math., 136(3):203-236, 2002.

[4] F. Bayart, A. Defant, L. Frerick, M. Maestre, and P. Sevilla-Peris. Monomial series expansion of $H_{p}$-functions and multipliers of $\mathcal{H}_{p}$-Dirichlet series. arXiv:1405. 7205, 2014.

[5] F. Bayart, D. Pellegrino, and J. B. Seoane-Sepúlveda. The Bohr radius of the $n$-dimensional polydisk is equivalent to $\sqrt{(\log n) / n}$. Adv. Math., 264:726-746, 2014.

[6] F. Bayart, H. Queffélec, and K. Seip. Approximation numbers of composition operators on $H_{p}$ spaces of Dirichlet series. arXiv:1406.0445, 2014. 
[7] H. F. Bohnenblust and E. Hille. On the absolute convergence of Dirichlet series. Ann. of Math. (2), 32(3):600-622, 1931.

[8] H. Bohr. Über die Bedeutung der Potenzreihen unendlich vieler Variablen in der Theorie der Dirichlet-schen Reihen $\sum \frac{a_{n}}{n^{s}}$. Nachr. Ges. Wiss. Göttingen, Math. Phys. Kl., pages 441-488, 1913.

[9] D. Carando, A. Defant, and P. Sevilla-Peris. Bohr's absolute convergence problem for $\mathcal{H}_{p}$-Dirichlet series in Banach spaces. Anal. PDE, 7(2):513-527, 2014.

[10] D. Carando, A. Defant, and P. Sevilla-Peris. Some polynomial versions of cotype and applications. arXiv:1503.00850, 2015.

[11] B. J. Cole and T. W. Gamelin. Representing measures and Hardy spaces for the infinite polydisk algebra. Proc. London Math. Soc. (3), 53(1):112-142, 1986.

[12] R. de la Bretèche. Sur l'ordre de grandeur des polynômes de Dirichlet. Acta Arith., 134(2):141$148,2008$.

[13] A. Defant, L. Frerick, J. Ortega-Cerdà, M. Ounaïes, and K. Seip. The Bohnenblust-Hille inequality for homogeneous polynomials is hypercontractive. Ann. of Math. (2), 174(1):485-497, 2011.

[14] A. Defant, D. García, M. Maestre, and D. Pérez-García. Bohr's strip for vector valued Dirichlet series. Math. Ann., 342(3):533-555, 2008.

[15] A. Defant, D. García, M. Maestre, and P. Sevilla-Peris. Bohr's fundamental theorem for vectorvalued Hardy-Dirichlet spaces. preprint, 2014.

[16] A. Defant, M. Maestre, and U. Schwarting. Bohr radii of vector valued holomorphic functions. Adv. Math., 231(5):2837-2857, 2012.

[17] A. Defant and A. Pérez. The vector-valued Bohr transform. preprint, 2014.

[18] A. Defant, U. Schwarting, and P. Sevilla-Peris. Estimates for vector valued Dirichlet polynomials. Monatsh. Math., 175(1):89-116, 2014.

[19] J. Diestel, H. Jarchow, and A. Tonge. Absolutely summing operators, volume 43 of Cambridge Studies in Advanced Mathematics. Cambridge University Press, Cambridge, 1995.

[20] P. Hartman. On Dirichlet series involving random coefficients. Amer. J. Math., 61:955-964, 1939.

[21] H. Hedenmalm, P. Lindqvist, and K. Seip. A Hilbert space of Dirichlet series and systems of dilated functions in $L^{2}(0,1)$. Duke Math. J., 86(1):1-37, 1997.

[22] A. Hildebrand, and G. Tenenbaum. Integers without large prime factors. J. Théor. Nombres Bordeaux, 5 (2):411-484, 1993.

[23] S. V. Konyagin and H. Queffélec. The translation $\frac{1}{2}$ in the theory of Dirichlet series. Real Anal. Exchange, 27(1):155-175, 2001/02.

[24] J. Lindenstrauss and L. Tzafriri. Classical Banach spaces. II, volume 97 of Ergebnisse der Mathematik und ihrer Grenzgebiete [Results in Mathematics and Related Areas]. Springer-Verlag, Berlin, 1979. Function spaces.

[25] B. Maurizi and H. Queffélec. Some remarks on the algebra of bounded Dirichlet series. J. Fourier Anal. Appl., 16(5):676-692, 2010.

[26] H. Queffélec. H. Bohr's vision of ordinary Dirichlet series; old and new results. J. Anal., 3:43-60, 1995.

[27] H. Queffélec and M. Queffélec. Diophantine approximation and Dirichlet series, volume 2 of Harish-Chandra Research Institute Lecture Notes. Hindustan Book Agency, New Delhi, 2013. 
[28] G. Tenenbaum. Introduction to analytic and probabilistic number theory, volume 46 of Cambridge Studies in Advanced Mathematics. Cambridge University Press, Cambridge, 1995. Translated from the second French edition (1995) by C. B. Thomas. 